\title{
Measurement of radiative Bhabha and quasi-real Compton scattering
}

\section{L3 Collaboration}

M. Acciarri ${ }^{\text {ab }}$, O. Adriani ${ }^{\mathrm{q}}$, M. Aguilar-Benitez ${ }^{\text {aa }}$, S. Ahlen ${ }^{\text {, }}$ J. Alcaraz ${ }^{\text {aa }}$, G. Alemanni w, J. Allaby ', A. Aloisio ad, M.G. Alviggi ad, G. Ambrosi ', H. Anderhub aw, V.P. Andreev ${ }^{\text {g,al }}$, T. Angelescu ${ }^{\mathrm{n}}$, F. Anselmo ${ }^{\mathrm{j}}$, A. Arefiev ${ }^{\text {ac }}$, T. Azemoon ${ }^{\text {c }}$, T. Aziz ${ }^{\text {k }}$, P. Bagnaia ${ }^{\text {ak }}$, L. Baksay ${ }^{\text {ar }}$, S. Banerjee ${ }^{\text {k }}$, Sw. Banerjee ${ }^{\text {, }}$, K. Banicz ${ }^{\text {at }}$, A. Barczyk ${ }^{a w, a u}$, R. Barillère ${ }^{r}$, L. Barone ${ }^{a k}$, P. Bartalini ${ }^{w}$, A. Baschirotto ${ }^{\text {ab }}$, M. Basile ${ }^{j}$, R. Battiston ${ }^{\text {ah }}$, A. Bay ${ }^{\text {w }}$, F. Becattini ${ }^{\mathrm{q}}$, U. Becker ${ }^{\mathrm{p}}$, F. Behner ${ }^{\text {aw }}$, J. Berdugo ${ }^{\text {aa }}$, P. Berges ${ }^{\mathrm{p}}$, B. Bertucci ${ }^{\text {ah }}$, B.L. Betev ${ }^{\text {aw }}$, S. Bhattacharya ${ }^{k}$, M. Biasini ${ }^{\text {ah }}$, A. Biland ${ }^{\text {aw }}$, G.M. Bilei ${ }^{\text {ah }}$, J.J. Blaising ${ }^{d}$, S.C. Blyth ${ }^{\text {ai }}$, G.J. Bobbink ${ }^{\text {b }}$, R. Bock ${ }^{\text {a }}$, A. Böhm ${ }^{\text {a }}$, L. Boldizsar ${ }^{\circ}$, B. Borgia ${ }^{\text {rak }}$, D. Bourilkov ${ }^{\text {aw }}$, M. Bourquin ${ }^{\mathrm{t}}$, S. Braccini ${ }^{\mathrm{t}}$, J.G. Branson ${ }^{\text {an }}$, V. Brigljevic ${ }^{\text {aw }}$, I.C. Brock ${ }^{\text {ai }}$, A. Buffini ${ }^{\mathrm{q}}$, A. Buijs ${ }^{\text {as }}$, J.D. Burger ${ }^{\text {p }}$, W.J. Burger ${ }^{\text {ah }}$, J. Busenitz ${ }^{\text {ar }}$, A. Button ${ }^{\text {c }}$, X.D. Cai ${ }^{\text {p }}$, M. Campanelli ${ }^{\text {aw }}$, M. Capell ${ }^{\text {p }}$, G. Cara Romeo ${ }^{j}$, G. Carlino ${ }^{\text {ad }}$, A.M. Cartacci ${ }^{\text {q }}$, J. Casaus ${ }^{\text {aa }}$, G. Castellini ${ }^{\text {q }}$, F. Cavallari ${ }^{\text {ak }}$, N. Cavallo ${ }^{\text {ad }}$, C. Cecchi ${ }^{t}$, M. Cerrada ${ }^{\text {aa }}$, F. Cesaroni ${ }^{\mathrm{x}}$, M. Chamizo ${ }^{\text {aa }}$, Y.H. Chang ${ }^{\text {ay }}$, U.K. Chaturvedi ${ }^{\text {s }}$, M. Chemarin ${ }^{\mathrm{z}}$, A. Chen ${ }^{\text {ay }}$, G. Chen ${ }^{\mathrm{h}}$, G.M. Chen ", H.F. Chen ", H.S. Chen ${ }^{\text {h }}$, X. Chereau ${ }^{\text {d }}$, G. Chiefari ${ }^{\text {ad, }}$ C.Y. Chien ${ }^{\mathrm{e}}$, L. Cifarelli ${ }^{\mathrm{am}}$, F. Cindolo $^{\mathrm{j}}$, C. Civinini ${ }^{\mathrm{q}}$, I. Clare ${ }^{\mathrm{p}}$, R. Clare ${ }^{\mathrm{p}}$, G. Coignet ${ }^{\mathrm{d}}$, A.P. Colijn ${ }^{\mathrm{b}}$, N. Colino ${ }^{\text {aa }}$, S. Costantini ${ }^{\mathrm{i}}$, F. Cotorobai ${ }^{\mathrm{n}}$, B. de la Cruz ${ }^{\text {aa }}$, A. Csilling ${ }^{\circ}$, T.S. Dai ${ }^{\text {p }}$, R. D'Alessandro ${ }^{q}$, R. de Asmundis ad, A. Degré ${ }^{d}$, K. Deiters ${ }^{\text {au }}$, D. della Volpe ${ }^{\text {ad }}$, P. Denes ${ }^{\text {aj }}$, F. DeNotaristefani ${ }^{\text {ak}}$, M. Diemoz ${ }^{\text {ak }}$, D. van Dierendonck ${ }^{b}$, F. Di Lodovico ${ }^{\text {aw }}$, C. Dionisi ${ }^{\text {rak }}$, M. Dittmar ${ }^{\text {aw }}$, A. Dominguez ${ }^{\text {an }}$, A. Doria ${ }^{\text {ad }}$, M.T. Dova ${ }^{\text {s,1 }}$, D. Duchesneau ${ }^{\text {d, }}$ P. Duinker ${ }^{\text {b }}$, I. Duran ${ }^{\text {ao }}$, S. Easo ${ }^{\text {ah }}$, H. El Mamouni ${ }^{\text {z }}$, A. Engler ai, F.J. Eppling ${ }^{\text {p }}$, F.C. Erné ${ }^{\text {b }}$, J.P. Ernenwein ${ }^{\mathrm{z}}$, P. Extermann ${ }^{\mathrm{t}}$, M. Fabre ${ }^{\text {au }}$, R. Faccini ${ }^{\text {ak }}$, M.A. Falagan ${ }^{\text {aa }}$, S. Falciano ${ }^{\text {ak }}$, A. Favara ${ }^{q}$, J. Fay ${ }^{\text {z }}$, O. Fedin ${ }^{\text {al }}$, M. Felcini ${ }^{\text {aw }}$, T. Ferguson ${ }^{\text {ai }}$, F. Ferroni ${ }^{\text {ak }}$, H. Fesefeldt a, E. Fiandrini ${ }^{\text {ah }}$, J.H. Field ${ }^{\text {t }}$,

F. Filthaut ${ }^{\mathrm{r}}$, P.H. Fisher ${ }^{\mathrm{p}}$, I. Fisk ${ }^{\text {an }}$, G. Forconi ${ }^{\mathrm{p}}$, L. Fredj ${ }^{\mathrm{t}}$, K. Freudenreich ${ }^{\text {aw }}$, 
C. Furetta $^{\text {ab }}$, Yu. Galaktionov ${ }^{a c, p}$, S.N. Ganguli k ${ }^{\text {, P. Garcia-Abia }}{ }^{\text {f }}$, M. Gataullin ${ }^{\text {ag }}$, S.S. Gau ${ }^{\mathrm{m}}$, S. Gentile ${ }^{\text {ak }}$, N. Gheordanescu ${ }^{\text {n, S. Giagu }}{ }^{\text {ak }}$, S. Goldfarb ${ }^{\text {w, }}$ J. Goldstein ', Z.F. Gong ", A. Gougas e, G. Gratta ${ }^{\text {ag }}$, M.W. Gruenewald ${ }^{\text {i }}$, R. van Gulik b, V.K. Gupta aj, A. Gurtu ${ }^{\text {k }}$, L.J. Gutay ${ }^{\text {at }}$, D. Haas ${ }^{\text {f }}$, B. Hartmann ${ }^{\text {a }}$, A. Hasan ${ }^{\text {ae }}$, D. Hatzifotiadou ${ }^{j}$, T. Hebbeker ${ }^{i}$, A. Hervé ${ }^{r}$, P. Hidas ${ }^{\circ}$, J. Hirschfelder ${ }^{\text {ai }}$, W.C. van Hoek ${ }^{\text {af }}$, H. Hofer ${ }^{\text {aw }}$, H. Hoorani ${ }^{\text {ai }}$, S.R. Hou ${ }^{\text {ay }}$, G. Hu ${ }^{\text {e }}$, I. Iashvili ${ }^{\text {av }}$, B.N. Jin ${ }^{\text {h }}$, L.W. Jones ${ }^{c}$, P. de Jong ${ }^{r}$, I. Josa-Mutuberria ${ }^{\text {aa }}$, R.A. Khan ${ }^{\text {s }}$, D. Kamrad av ${ }^{\text {, J.S. Kapustinsky }}{ }^{\mathrm{y}}$, M. Kaur ${ }^{\text {s,2 }}$, M.N. Kienzle-Focacci ${ }^{\mathrm{t}}$, D. Kim ${ }^{\text {ak }}$, D.H. Kim ${ }^{\text {aq }}$, J.K. Kim ${ }^{\text {aq }}$, S.C. Kim ${ }^{\text {aq }}$, W.W. Kinnison ${ }^{\text {y }}$, A. Kirkby ${ }^{\text {ag }}$, D. Kirkby ${ }^{\text {ag }}$, J. Kirkby ${ }^{\text {r }}$, D. Kiss ${ }^{\circ}$, W. Kittel ${ }^{\text {af }}$, A. Klimentov ${ }^{\text {pac }}$, A.C. König af ${ }^{\text {, }}$ A. Kopp ${ }^{\text {av }}$, I. Korolko ${ }^{\text {ac }}$, V. Koutsenko ${ }^{\text {pac }}{ }^{\text {ac }}$ R.W. Kraemer ${ }^{\text {ai }}$, W. Krenz ${ }^{\text {a }}$, A. Kunin ${ }^{\text {pac }}$, P. Lacentre ${ }^{\text {av }, 1,3}$, P. Ladron de Guevara ${ }^{\text {aa }}$, I. Laktineh ${ }^{\mathrm{z}}$, G. Landi ${ }^{\mathrm{q}}$, C. Lapoint $^{\mathrm{p}}$, K. Lassila-Perini ${ }^{\text {aw }}$, P. Laurikainen ${ }^{\mathrm{v}}$, A. Lavorato ${ }^{\mathrm{am}}$, M. Lebeau ${ }^{\mathrm{r}}$, A. Lebedev ${ }^{p}$, P. Lebrun ${ }^{z}$, P. Lecomte ${ }^{\text {aw }}$, P. Lecoq ${ }^{r}$, P. Le Coultre ${ }^{\text {aw }}$, H.J. Lee ${ }^{\text {i }}$, J.M. Le Goff ${ }^{\mathrm{r}}$, R. Leiste ${ }^{\text {av }}$, E. Leonardi ${ }^{\text {ak }}$, P. Levtchenko ${ }^{\text {al }}$, C. Li ${ }^{\text {u }}$, C.H. Lin ${ }^{\text {ay }}$, W.T. Lin ${ }^{\text {ay }}$, F.L. Linde ${ }^{\text {b,r }}$, L. Lista ${ }^{\text {ad }}$, Z.A. Liu ${ }^{\text {h }}$, W. Lohmann ${ }^{\text {av }}$, E. Longo ${ }^{\text {ak }}$, W. Lu ${ }^{\text {ag }}$, Y.S. Lu ${ }^{\text {h }}$, K. Lübelsmeyer ${ }^{\text {a }}$, C. Luci rak ${ }^{\text {,ak }}$ D. Luckey ${ }^{p}$, L. Luminari ak, W. Lustermann ${ }^{\text {aw }}$, W.G. Ma ${ }^{\text {u }}$, M. Maity ${ }^{\text {k}}$, G. Majumder ${ }^{\text {k }}$, L. Malgeri ${ }^{\mathrm{r}}$, A. Malinin ${ }^{\text {ac }}$, C. Maña aa , D. Mangeol af ${ }^{\text {a }}$, P. Marchesini ${ }^{\text {aw }}$, G. Marian ${ }^{\text {ar,4 }}$, A. Marin ${ }^{1}$, J.P. Martin ${ }^{\mathrm{z}}$, F. Marzano ${ }^{\mathrm{ak}}$, G.G.G. Massaro ${ }^{\mathrm{b}}$, K. Mazumdar ${ }^{\mathrm{k}}$, R.R. McNeil ${ }^{\mathrm{g}}$,

S. Mele ${ }^{\mathrm{r}}$, L. Merola ${ }^{\text {ad }}$, M. Meschini ${ }^{\mathrm{q}}$, W.J. Metzger af ${ }^{\text {, M. Mon der Mey }}{ }^{\mathrm{a}}$, D. Migani ${ }^{\mathrm{j}}$, A. Mihul ${ }^{\mathrm{n}}$, A.J.W. van Mil af ${ }^{\mathrm{p}}$, H. Milcent ${ }^{\mathrm{r}}$, G. Mirabelli ${ }^{\mathrm{ak}}$, J. Mnich ${ }^{\mathrm{r}}$, P. Molnar ${ }^{\text {i }}$, B. Monteleoni q , R. Moore ${ }^{\text {c }}$, T. Moulik k, R. Mount ${ }^{\text {ag }}$, F. Muheim ', A.J.M. Muijs ${ }^{b}$, S. Nahn ${ }^{p}$, M. Napolitano ${ }^{\text {ad }}$, F. Nessi-Tedaldi aw ${ }^{\text {aw }}$ H. Newman ${ }^{\text {ag }}$, T. Niessen ${ }^{\text {a }}$, A. Nippe ${ }^{\text {w }}$, A. Nisati ${ }^{\text {ak }}$, H. Nowak ${ }^{\text {av }}$, Y.D. Oh ${ }^{\text {aq }}$, G. Organtini ${ }^{\text {ak }}$,

R. Ostonen ${ }^{\text {, }, ~ S . ~ P a l i t ~}{ }^{\mathrm{m}}$, C. Palomares ${ }^{\text {aa }}$, D. Pandoulas ${ }^{\mathrm{a}}$, S. Paoletti ${ }^{\mathrm{ak}, \mathrm{r}}$, P. Paolucci ad ${ }^{a}$, H.K. Park ${ }^{\text {a }}$, I.H. Park ${ }^{\text {aq }}$, G. Pascale ${ }^{\text {ak }}$, G. Passaleva ${ }^{\text {r }}$, S. Patricelli ad, T. Paul ${ }^{\mathrm{m}}$, M. Pauluzzi ah ${ }^{\text {a }}$ C. Paus ${ }^{\mathrm{r}}$, F. Pauss ${ }^{\text {aw }}$, D. Peach ${ }^{\mathrm{r}}$, Y.J. Pei ${ }^{\mathrm{a}}$, S. Pensotti ${ }^{\text {ab }}$, D. Perret-Gallix ${ }^{\text {d }}$, B. Petersen ${ }^{\text {af }}$, S. Petrak ${ }^{i}$, A. Pevsner ${ }^{\text {e, }}$, D. Piccolo ${ }^{\text {ad }}$, M. Pieri ${ }^{\mathrm{q}}$, P.A. Piroué ${ }^{\mathrm{aj}}$, E. Pistolesi ${ }^{\text {ab }}$, V. Plyaskin ${ }^{\mathrm{ac}}$, M. Pohl ${ }^{\mathrm{aw}}$, V. Pojidaev ${ }^{a c, q}$, H. Postema ${ }^{p}$, J. Pothier ${ }^{r}$, N. Produit ${ }^{t}$, D. Prokofiev ${ }^{a l}$, J. Quartieri ${ }^{\text {am }}$, G. Rahal-Callot ${ }^{\text {aw }}$, N. Raja ${ }^{\text {k, P.G. Rancoita }}{ }^{\text {ab }}$, M. Rattaggi ${ }^{\text {ab }}$, G. Raven ${ }^{\text {an }}$, P. Razis ${ }^{\text {ae }}$, D. Ren ${ }^{\text {aw }}$, M. Rescigno ak, S. Reucroft ${ }^{\mathrm{m}}$, T. van Rhee ${ }^{\text {as }}$, S. Riemann ${ }^{\text {av }}$, K. Riles ${ }^{\text {c }}$, A. Robohm ${ }^{\text {aw }}$, J. Rodin ${ }^{\text {ar }}$, B.P. Roe ${ }^{\text {c }}$, L. Romero ${ }^{\text {aa }}$, S. Rosier-Lees ${ }^{\text {d }, ~ S . ~ R o t h ~}{ }^{\text {a }}$, J.A. Rubio ${ }^{\text {r }}$, D. Ruschmeier ${ }^{\mathrm{i}}$, H. Rykaczewski ${ }^{\text {aw }}$, J. Salicio ${ }^{r}$, E. Sanchez ${ }^{\text {aa }}$, M.P. Sanders af , M.E. Sarakinos ${ }^{\text {v }}$, C. Schäfer ${ }^{\text {a }}$, V. Schegelsky ${ }^{\text {al }}$, S. Schmidt-Kaerst ${ }^{\text {a }}$, D. Schmitz ${ }^{\text {a }}$, N. Scholz ${ }^{\text {aw }}$, H. Schopper ${ }^{\text {ax }}$, D.J. Schotanus ${ }^{\text {af }}$, J. Schwenke ${ }^{\text {a }}$ G. Schwering a , C. Sciacca ${ }^{\text {ad }}$, 
D. Sciarrino ${ }^{t}$, L. Servoli ah, S. Shevchenko ${ }^{\text {ag }}$, N. Shivarov ap, V. Shoutko ${ }^{\text {ac }}$, J. Shukla ${ }^{y}$, E. Shumilov ${ }^{\text {ac }}$, A. Shvorob ${ }^{\text {ag }}$, T. Siedenburg ${ }^{a}$, D. Son ${ }^{\text {aq }}$, B. Smith ${ }^{p}$, P. Spillantini ${ }^{\mathrm{q}}$, M. Steuer ${ }^{\mathrm{p}}$, D.P. Stickland ${ }^{\text {aj }}$, A. Stone ${ }^{\mathrm{g}}$, H. Stone ${ }^{\text {aj, }}$, B. Stoyanov ap, A. Straessner ${ }^{\text {a }}$, K. Sudhakar ${ }^{\text {k, G. Sultanov s, L.Z. Sun " }}$, G.F. Susinno ${ }^{\mathrm{t}}$, H. Suter ${ }^{\text {aw }}$, J.D. Swain ${ }^{\mathrm{s}}$, X.W. Tang ${ }^{\mathrm{h}}$, L. Tauscher ${ }^{\mathrm{f}}$, L. Taylor ${ }^{\mathrm{m}}$, C. Timmermans af , Samuel C.C. Ting ${ }^{\mathrm{p}}$, S.M. Ting ${ }^{\mathrm{p}}$, S.C. Tonwar ${ }^{\mathrm{k}}$, J. Tóth ${ }^{\circ}$, C. Tully ${ }^{\text {aj }}$, K.L. Tung ${ }^{h}$, Y. Uchida ${ }^{p}$, J. Ulbricht ${ }^{\text {aw }}$, E. Valente ${ }^{\text {ak }}$, G. Vesztergombi ${ }^{\circ}$, I. Vetlitsky ${ }^{\text {ac }}$, G. Viertel ${ }^{\text {aw }}$, M. Vivargent ${ }^{d}$, S. Vlachos ${ }^{\text {f }}$, H. Vogel ${ }^{\text {ai }}$, H. Vogt ${ }^{\text {av }}$, I. Vorobiev ${ }^{\text {r,ac }}$, A.A. Vorobyov ${ }^{\text {al }}$, A. Vorvolakos ${ }^{\text {ae }}$,

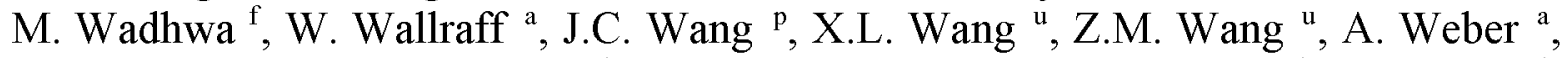
S.X. Wu ${ }^{\text {p }, ~ S . ~ W y n h o f f ~ a ~, ~ J . ~ X u ~}{ }^{1}$, Z.Z. Xu ${ }^{\text {u }, ~ B . Z . ~ Y a n g ~ " ~, ~ C . G . ~ Y a n g ~}{ }^{\text {h }}$, H.J. Yang ${ }^{\text {h }}$, M. Yang ${ }^{\text {h}}$, J.B. Ye ${ }^{\text {u }}$, S.C. Yeh ${ }^{\text {az }}$, J.M. You ${ }^{\text {ai }}$, An. Zalite ${ }^{\text {al }}$, Yu. Zalite ${ }^{\text {al }}$, P. Zemp ${ }^{\text {aw }}$, Y. Zeng a , Z.P. Zhang " , B. Zhou ${ }^{1}$, G.Y. Zhu ${ }^{\text {h }}$, R.Y. Zhu ${ }^{\text {ag }}$, A. Zichichi ${ }^{\mathrm{j}, \mathrm{r}, \mathrm{s}}$, F. Ziegler ${ }^{\text {av }}$, G. Zilizi ${ }^{\text {ar, } 4}$

a I. Physikalisches Institut, RWTH, D-52056 Aachen, Germany, and III. Physikalisches Institut, RWTH, D-52056 Aachen, Germany ${ }^{5}$

${ }^{\mathrm{b}}$ National Institute for High Energy Physics, NIKHEF, and University of Amsterdam, NL-1009 DB Amsterdam, Netherlands

c University of Michigan, Ann Arbor, MI 48109, USA

'Laboratoire d'Annecy-le-Vieux de Physique des Particules, LAPP, IN2P3-CNRS, BP 110, F-74941 Annecy-le-Vieux CEDEX, France e Johns Hopkins University, Baltimore, MD 21218, USA

$\mathrm{f}$ Institute of Physics, University of Basel, $\mathrm{CH}-4056$ Basel, Switzerland

${ }^{\mathrm{g}}$ Louisiana State University, Baton Rouge, LA 70803, USA

${ }^{\mathrm{h}}$ Institute of High Energy Physics, IHEP, 100039 Beijing, China ${ }^{6}$

${ }^{\mathrm{i}}$ Humboldt University, D-10099 Berlin, Germany ${ }^{5}$

${ }^{j}$ University of Bologna and INFN-Sezione di Bologna, I-40126 Bologna, Italy

${ }^{\mathrm{k}}$ Tata Institute of Fundamental Research, Bombay 400005 , India

${ }^{1}$ Boston University, Boston, MA 02215, USA

${ }^{\mathrm{m}}$ Northeastern University, Boston, MA 02115, USA

${ }^{\mathrm{n}}$ Institute of Atomic Physics and University of Bucharest, R-76900 Bucharest, Romania

${ }^{\circ}$ Central Research Institute for Physics of the Hungarian Academy of Sciences, H-1525 Budapest 114, Hungary ${ }^{7}$

${ }^{\mathrm{p}}$ Massachusetts Institute of Technology, Cambridge, MA 02139, USA

${ }^{\mathrm{q}}$ INFN Sezione di Firenze and University of Florence, I-50125 Florence, Italy

${ }^{\mathrm{r}}$ European Laboratory for Particle Physics, CERN, CH-1211 Geneva 23, Switzerland

"World Laboratory, FBLJA Project, CH-1211 Geneva 23, Switzerland

${ }^{\mathrm{t}}$ University of Geneva, CH-1211 Geneva 4, Switzerland

"Chinese University of Science and Technology, USTC, Hefei, Anhui 230 029, China ${ }^{6}$

" SEFT, Research Institute for High Energy Physics, P.O. Box 9, SF-00014 Helsinki, Finland

${ }^{\mathrm{w}}$ University of Lausanne, CH-1015 Lausanne, Switzerland

${ }^{x}$ INFN-Sezione di Lecce and Universitá Degli Studi di Lecce, I-73I00 Lecce, Italy

' Los Alamos National Laboratory, Los Alamos, NM 87544, USA

${ }^{\mathrm{z}}$ Institut de Physique Nucléaire de Lyon, IN2P3-CNRS, Université Claude Bernard, F-69622 Villeurbanne, France

${ }^{\text {aa }}$ Centro de Investigaciones Energeticas, Medioambientales y Tecnologicas, CIEMAT, E-28040 Madrid, Spain ${ }^{8}$

ab INFN-Sezione di Milano, I-20133 Milan, Italy

ac Institute of Theoretical and Experimental Physics, ITEP, Moscow, Russia

${ }^{\text {ad }}$ INFN-Sezione di Napoli and University of Naples, I-80125 Naples, Italy

ae Department of Natural Sciences, University of Cyprus, Nicosia, Cyprus

af University of Nijmegen and NIKHEF, NL-6525 ED Nijmegen, Netherlands

ag California Institute of Technology, Pasadena, CA 91125, USA

ah INFN-Sezione di Perugia and Universitá Degli Siudi di Perugia, I-06100 Perugia, Italy

ai Carnegie Mellon University, Pittsburgh, PA 15213, USA

aj Princeton University, Princeton, NJ 08544, USA 


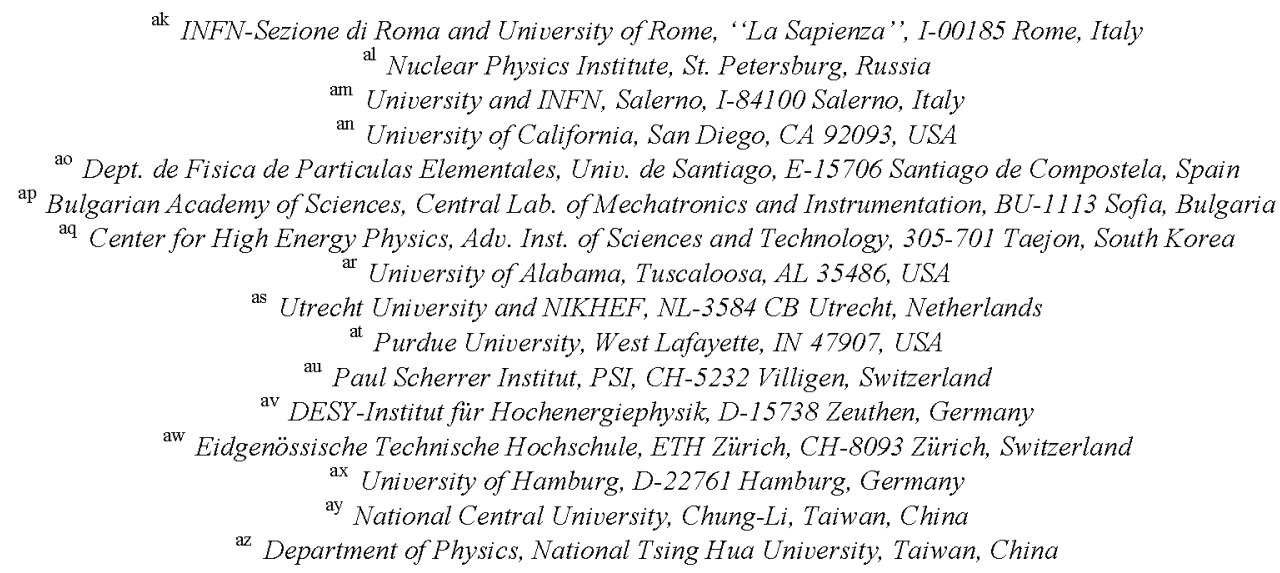

Received 29 June 1998

Editor: K. Winter

\begin{abstract}
We report on a study of radiative Bhabha and quasi-real Compton scattering at centre-of-mass energies between $50 \mathrm{GeV}$ and $170 \mathrm{GeV}$, and $20 \mathrm{GeV}$ and $140 \mathrm{GeV}$, respectively, using the $\mathrm{L} 3$ detector at LEP. The analysis is based on data corresponding to an integrated luminosity of $232.2 \mathrm{pb}^{-1}$. A total of 2856 radiative Bhabha and 4641 Compton scattering events are collected. Total and differential cross sections for both reactions are presented and found to be in good agreement with QED expectations. Our measurement of Compton scattering at the highest energies obtained so far is used to derive exclusion limits on the coupling $\lambda$ for the on-shell production of an excited electron $\mathrm{e}^{\star}$ decaying into a $\gamma \mathrm{e}$ pair in the mass range $20 \mathrm{GeV}<m_{\mathrm{e}^{\star}}<170 \mathrm{GeV}$. (C) 1998 Published by Elsevier Science B.V. All rights reserved.
\end{abstract}

\section{Introduction}

At LEP, Bhabha scattering has been measured at centre-of-mass energies, $\sqrt{s}$, between $88 \mathrm{GeV}$ and $172 \mathrm{GeV}[1]$. The energy range from $12 \mathrm{GeV}$ to 60 $\mathrm{GeV}$ is covered by data from experiments at PEP,

\footnotetext{
${ }^{1}$ Also supported by CONICET and Universidad Nacional de La Plata, CC 67, 1900 La Plata, Argentina.

${ }^{2}$ Also supported by Panjab University, Chandigarh-160014, India.

${ }^{3}$ Supported by Deutscher Akademischer Austauschdienst.

${ }^{4}$ Also supported by the Hungarian OTKA fund under contract numbers T22238 and T026178.

${ }^{5}$ Supported by the German Bundesministerium für Bildung, Wissenschaft, Forschung und Technologie.

${ }^{6}$ Supported by the National Natural Science Foundation of China.

${ }^{7}$ Supported by the Hungarian OTKA fund under contract numbers T019181, F023259 and T024011.

${ }^{8}$ Supported also by the Comisión Interministerial de Ciencia y Technología.
}

PETRA and TRISTAN [2]. The energy region between TRISTAN and LEP1 is not explored by direct measurements and the LEP2 energy points are sparse. However, all regions can be studied using events with hard initial-state radiation in which the fermion pair is produced at lower energies as has been performed for the reaction $\mathrm{e}^{+} \mathrm{e}^{-} \rightarrow \mu^{+} \mu^{-}(\gamma)$ [3].

Due to the effect of initial-state bremsstrahlung, lower energy beam electrons ${ }^{9}$ are produced accompanied by a high energy photon collinear to the beam direction. Either the electron or the photon can react with the other beam particle in a hard scattering process, resulting in either Bhabha or Compton scattering. Both reactions are illustrated in Fig. 1.

For Bhabha scattering with initial-state radiation the visible cross section is described by a convolution of the improved Born Bhabha cross section,

\footnotetext{
${ }^{9}$ If not mentioned otherwise, electron refers to both electron and positron.
} 

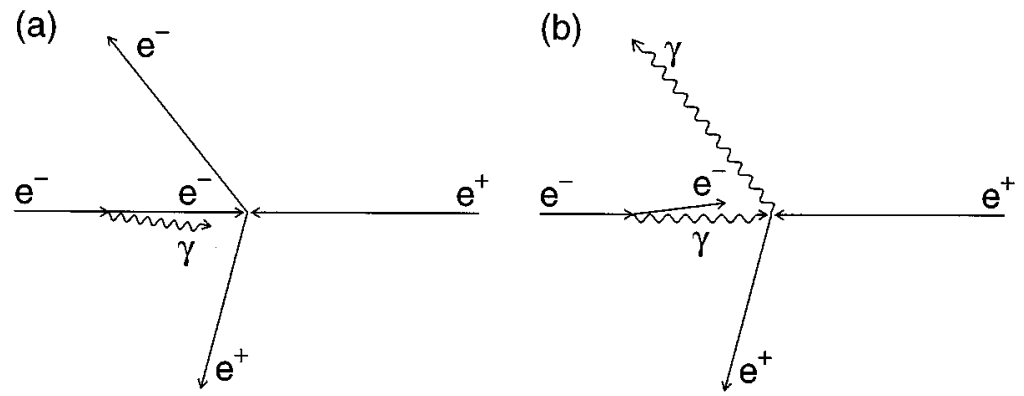

Fig. 1. Schematic representation of initial-state radiation at $\mathrm{e}^{+} \mathrm{e}^{-}$colliders for (a) radiative Bhabha scattering and (b) Compton scattering of quasi-real photons.

$\sigma_{\mathrm{ee}}^{\text {Born }}$, with a radiator function, $R\left(s^{\prime}, s\right)$, where $s^{\prime}$ is the reduced centre-of-mass energy squared [4]:

$\sigma(s)=\int_{4 m_{\mathrm{e}}^{2}}^{s} \mathrm{~d} s^{\prime} R\left(s^{\prime}, s\right) \sigma_{\mathrm{ee}}^{\mathrm{Born}}\left(s^{\prime}\right)$.

The unconvoluted Bhabha cross section at a reduced centre-of-mass energy is extracted by measuring the differential cross section of initial-state radiation:

$$
\frac{\mathrm{d} \sigma}{\mathrm{d} s^{\prime}}=R\left(s^{\prime}, s\right) \sigma_{\mathrm{ee}}^{\text {Born }}\left(s^{\prime}\right) .
$$

The cross section of quasi-real Compton scattering is described by a similar convolution given by [5]:

$$
\frac{\mathrm{d} \sigma}{\mathrm{d} s^{\prime}}=f_{\gamma \mathrm{e}}\left(s^{\prime}, s\right) \sigma_{\gamma \mathrm{e}}^{\mathrm{QED}}\left(s^{\prime}\right) .
$$

At lowest order, $\mathscr{O}\left(\alpha^{3}\right)$, the Compton cross section at Born-level, $\sigma_{\gamma \mathrm{e}}^{\mathrm{QED}}$, is folded with the equivalent photon spectrum, $f_{\gamma \mathrm{e}}$. The $\sqrt{s^{\prime}}$ spectrum of the selected events is then used to measure the unconvoluted Compton cross section at a reduced centre-ofmass energy.

In the following the analysis of the L3 data taken at the $Z$ resonance and at energies up to $183 \mathrm{GeV}$ is described and the measurement of the total and the differential cross sections of Bhabha and Compton scattering are presented.

The Compton scattering process in particular is sensitive to the production of an on-shell excited electron, $\mathrm{e}^{\star}$, decaying into an electron-photon pair. The measurements are used to derive upper limits on the $e^{\star}$ e $\gamma$ coupling for masses of the excited electron almost up to the centre-of-mass energy of the $e^{+} e^{-}$ collision, covering a mass range from $20 \mathrm{GeV}$ to 170 $\mathrm{GeV}$.

\section{Analysis techniques}

\subsection{Electron photon identification}

The $\mathrm{L} 3$ detector and its performance is described elsewhere [6]. The program BHAGENE3 [7] is used to simulate Bhabha scattering. The Monte Carlo prediction of Compton scattering is based on Monte Carlo events generated with the program TEEGG [8], taking into account corrections of order $\mathscr{O}\left(\alpha^{4}\right)$. The following Monte Carlo event generators are used to simulate the various background reactions: KORALZ [9] $\left(\mathrm{e}^{+} \mathrm{e}^{-} \rightarrow \tau^{+} \tau^{-}(\gamma)\right)$, GGG [10] $\left(\mathrm{e}^{+} \mathrm{e}^{-} \rightarrow\right.$ $\gamma \gamma(\gamma))$ and DIAG36 [11] $\left(\mathrm{e}^{+} \mathrm{e}^{-} \rightarrow \mathrm{e}^{+} \mathrm{e}^{-} \mathrm{e}^{+} \mathrm{e}^{-}\right)$. The response of the $\mathrm{L} 3$ detector is modelled with the GEANT [12] detector simulation program.

The identification of electrons and photons requires clusters in the electromagnetic calorimeter with an energy larger than $2 \mathrm{GeV}$ and consistent with an electromagnetic shower shape. A cluster is defined as an electron, if

- there is a reconstructed track within $5^{\circ}$ to the cluster direction in azimuthal angle $\phi$,

- or if more than $20 \%$ of the signals expected for an electron are present in the vertex chamber within a $1 \mathrm{~cm}$ wide road in the $r-\phi$ plane centred around the cluster direction.

A cluster is defined as a photon, if

- it is not identified as an electron,

- and the separation to the next electron candidate is larger than $8^{\circ}$. 

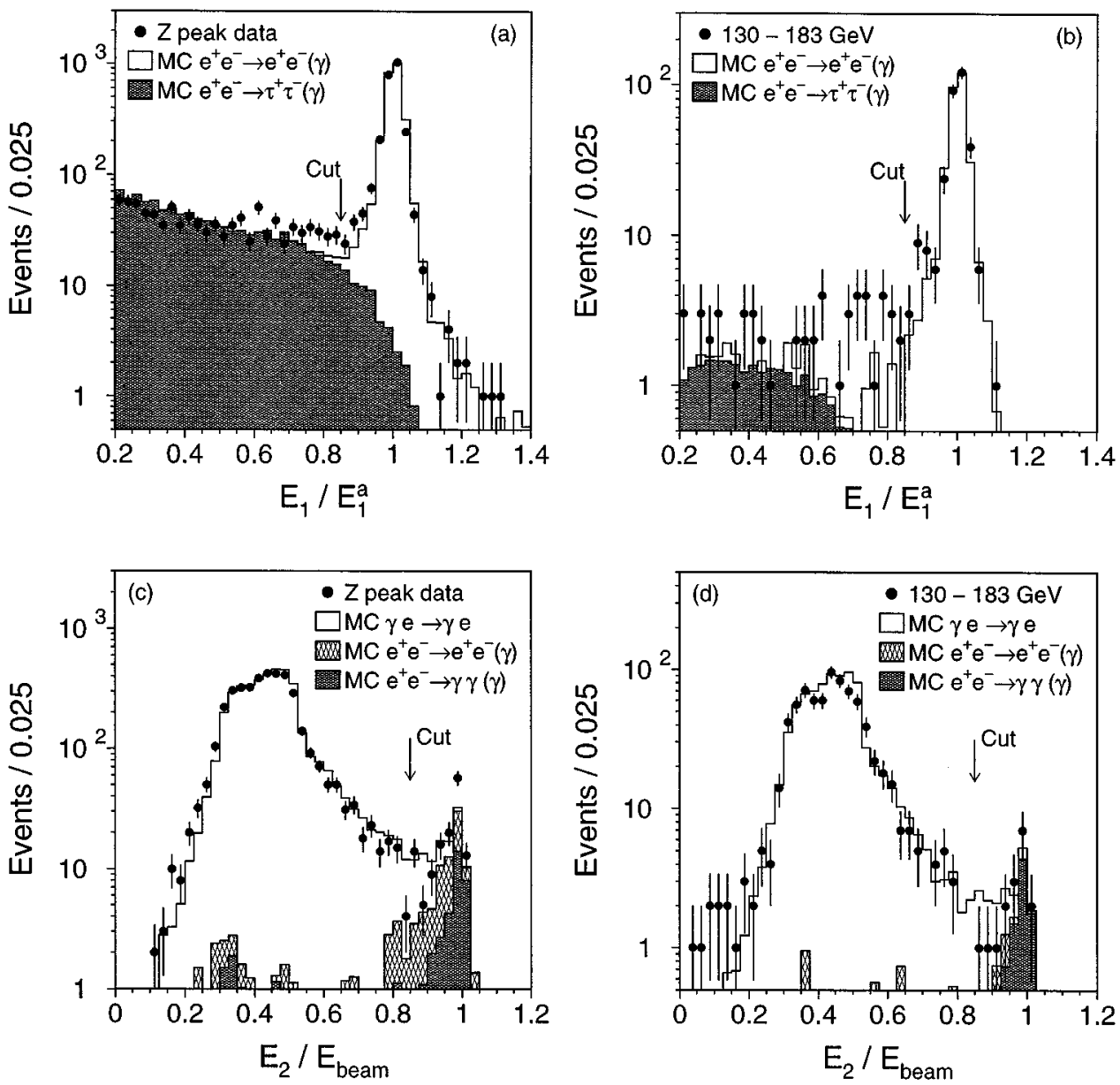

Fig. 2. The measured energy distributions of the most energetic electromagnetic cluster, $E_{1}$, normalized to the expected energy, $E_{1}^{a}$, calculated from the polar angles of the two clusters for radiative Bhabha scattering events obtained (a) at the Z peak and (b) in the LEP2 energy range as indicated. The measured energy distributions of the second electromagnetic cluster, $E_{2}$, normalized to the beam energy, $E_{\text {beam }}$, for Compton scattering events (c) at the $Z$ peak and (d) in the LEP2 energy range as indicated. Data and Monte Carlo simulation for signal and background are shown. All other selection cuts are applied.

\subsection{Reconstruction of the effective centre-of-mass energy}

For three-particle final states the particle momenta are obtained from the measured directions using energy and momentum conservation. Assuming that one undetected particle, either an electron or a photon, is radiated along the beam axis, its energy, $E_{\text {mis }}$, is given by the polar angles, $\theta_{1}$ and $\theta_{2}$, of the detected particles:

$E_{\mathrm{mis}}=\sqrt{s} \frac{\left|\sin \left(\theta_{1}+\theta_{2}\right)\right|}{\sin \theta_{1}+\sin \theta_{2}+\left|\sin \left(\theta_{1}+\theta_{2}\right)\right|}$.
In this case, the energies of the scattered particles, $E_{1}^{a}$ and $E_{2}^{a}$, are also determined by the polar angles:

$$
E_{1,2}^{a}=\sqrt{s} \frac{\sin \left(\theta_{2,1}\right)}{\sin \theta_{1}+\sin \theta_{2}+\left|\sin \left(\theta_{1}+\theta_{2}\right)\right|} .
$$

The effective centre-of-mass energy of the hard scattering process is calculated using the energy of the missing particle:

$s^{\prime}=s\left(1-2 \frac{E_{\mathrm{mis}}}{\sqrt{s}}\right)$. 
This procedure improves the resolution on $s^{\prime}$ as compared to the direct energy measurement. This is particularly important for data taken at $\sqrt{s} \approx m_{\mathrm{Z}}$ where the radiative part of the cross section is small.

The interpretation of the reconstructed $\sqrt{s^{\prime}}$ as the effective centre-of-mass energy after initial-state radiation is valid to the extent that interference effects between initial and final state radiation are negligible. The interference effects on Bhabha scattering are studied using the program TOPAZ0 [13]. They are found to be less than $0.05 \%$ of the total cross section for radiative events and are therefore negligible.

\section{Bhabha scattering with initial-state radiation}

\subsection{Event selection}

The selection of Bhabha scattering events requires two identified electrons inside the polar angular range of $|\cos \theta|<0.72$. The particle energies, $E$, measured in the calorimeter are compared to the values, $E^{a}$, reconstructed from the polar angles of the two electrons using Eq. (2). For the particle with the higher energy, $E_{1}$, a cut on the ratio, $E_{1} / E_{1}^{\alpha}>0.85$, is used to reduce background mainly coming from tau-pair

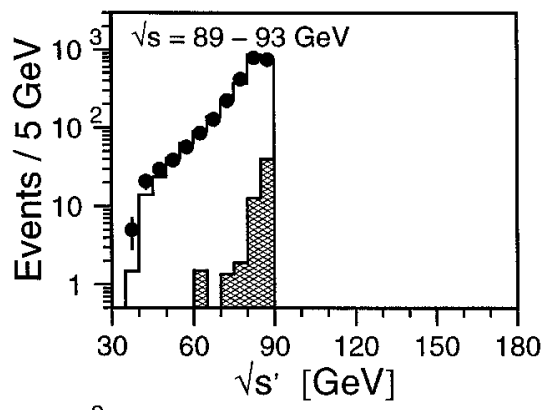

- Data

$\square \mathrm{MC} \mathrm{e}^{+} \mathrm{e}^{-} \rightarrow \mathrm{e}^{+} \mathrm{e}^{-}(\gamma)$


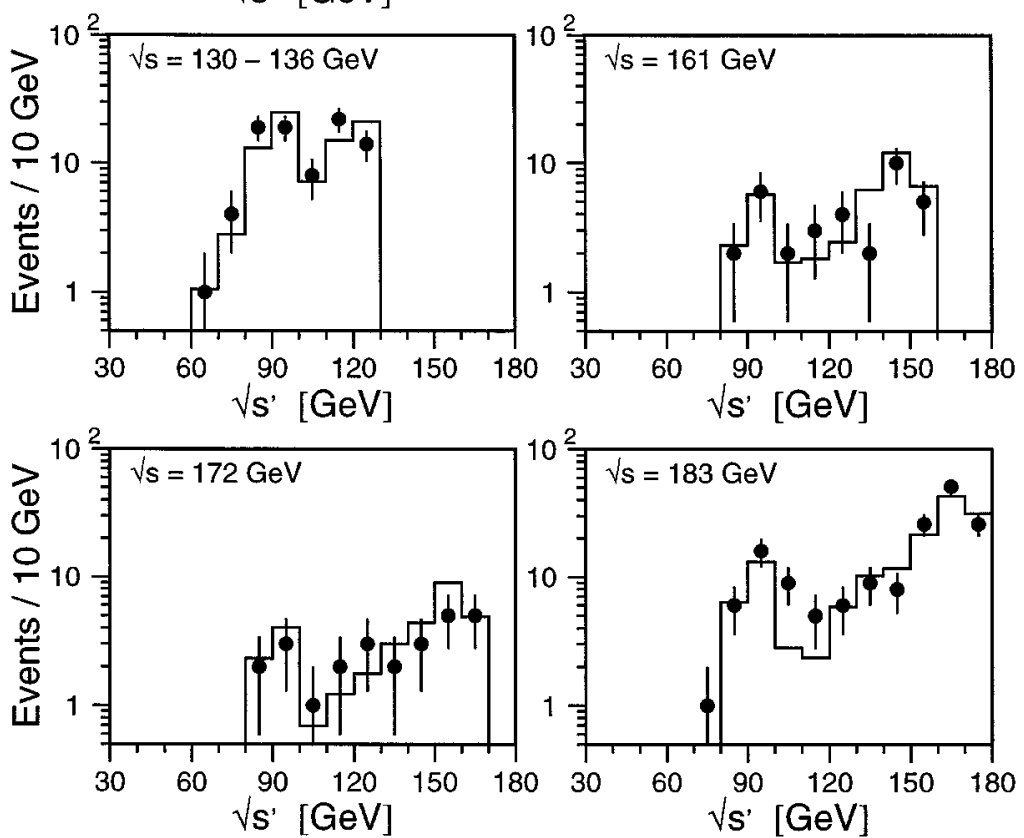

Fig. 3. The $\sqrt{s^{\prime}}$ spectra of Bhabha scattering for data and Monte Carlo simulation for signal and background are shown for different centre-of-mass energies. All selection cuts are applied. 
Table 1

Integrated luminosities recorded at the various centre-of-mass energies and the number of selected radiative Bhabha scattering events, $N_{\text {ee }}$, and quasi-real Compton scattering events, $N_{\gamma \mathrm{e}}$

\begin{tabular}{lcrr}
\hline$\sqrt{s}[\mathrm{GeV}]$ & $\int \mathscr{L} \mathrm{d} t\left[\mathrm{pb}^{-1}\right]$ & $N_{\mathrm{ee}}$ & \multicolumn{1}{c}{$N_{\gamma \mathrm{e}}$} \\
\hline $88-93$ & 143.9 & 2546 & 3882 \\
130 & 6.4 & 43 & 83 \\
136 & 6.0 & 44 & 81 \\
161 & 10.8 & 34 & 108 \\
172 & 10.1 & 26 & 82 \\
183 & 55.0 & 163 & 405 \\
\hline
\end{tabular}

production. Distributions of this ratio are shown in Fig. 2a and Fig. 2b with further cuts applied as described below.

For events with a three particle final-state, where one of the particles escapes along the beam axis, the two particles detected in the calorimeter should be back-to-back in the $r-\phi$ plane. Therefore, the two detected particles are required to have an acoplanarity angle of less than $3^{\circ}$.

Final state radiation can change the polar angle of the emitting electron and may cause a wrong reconstruction of the effective centre-of-mass energy. Events containing a third electromagnetic cluster with an energy larger than $2 \mathrm{GeV}$ and an angular separation of more than $8^{\circ}$ from any of the two identified electrons are therefore removed.
Events with a reduced energy, $\sqrt{s^{\prime} / s}<0.95$, are used for further analysis. The fraction of radiative Bhabha scattering events which pass this cut amounts to $2.0 \%$ for the data taken at the $Z$ resonance. This fraction varies from $18 \%$ to $14 \%$ for centre-of-mass energies between $130 \mathrm{GeV}$ and $183 \mathrm{GeV}$. Fig. 3 shows the resulting $\sqrt{s^{\prime}}$-distributions for Bhabha scattering at the different centre-of-mass energies. Good agreement between data and the Monte Carlo prediction is observed. The distributions are combined to determine the total cross sections at reduced centre-of-mass energies.

\subsection{Total cross sections}

The data sample was recorded in the years 1991 to 1997 and corresponds to a total integrated luminosity of $232.2 \mathrm{pb}^{-1}$, including a luminosity of $88.3 \mathrm{pb}^{-1}$ recorded at energies between $130 \mathrm{GeV}$ and $183 \mathrm{GeV}$. The luminosities recorded at the various centre-of-mass energies and the number of selected events are listed in Table 1. The sample contains in total 2856 radiative Bhabha events with a reconstructed $\sqrt{s^{\prime}}$ value between $35 \mathrm{GeV}$ and $175 \mathrm{GeV}$. They are used to measure cross sections in 11 bins of $\sqrt{s^{\prime}}$.

The Born cross section at an effective centre-ofmass energy is measured by scaling the theoretical

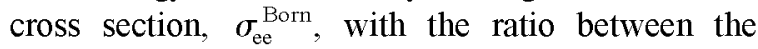

Table 2

Number of selected radiative Bhabha scattering events, $N_{\text {ee }}$, for the various ranges of $\sqrt{s^{\prime}}$ and their average $\left\langle\sqrt{s^{\prime}}\right\rangle$. The corresponding measured cross sections, $\sigma_{\mathrm{ee}}$, and their statistical and systematic errors are listed and compared to the Standard Model improved Born level cross sections, $\sigma_{\mathrm{ee}}^{\text {Bom }}$

\begin{tabular}{lcccc}
\hline$\sqrt{s^{\prime}}[\mathrm{GeV}]$ & $\left\langle\sqrt{s^{\prime}}\right\rangle[\mathrm{GeV}]$ & $N_{\mathrm{ee}}$ & $\sigma_{\mathrm{ee}} \pm(\mathrm{stat}) \pm(\mathrm{syst})[\mathrm{pb}]$ & $\sigma_{\mathrm{ee}}^{\text {Born }}[\mathrm{pb}]$ \\
\hline$<60$ & 52.0 & 152 & $449.4 \pm 35.1 \pm 20.2$ & 423.7 \\
$60-68$ & 64.5 & 153 & $258.3 \pm 23.5 \pm 12.5$ & 285.1 \\
$68-76$ & 72.5 & 335 & $231.5 \pm 13.2 \pm 7.4$ & 238.2 \\
$76-82$ & 79.2 & 594 & $235.5 \pm 9.3 \pm 5.5$ & 223.9 \\
$82-85$ & 83.7 & 575 & $224.0 \pm 10.6 \pm 5.9$ & 246.0 \\
$85-87$ & 86.1 & 622 & $300.0 \pm 12.6 \pm 8.1$ & 297.6 \\
$87-92$ & 88.3 & 169 & $483.9 \pm 37.1 \pm 13.2$ & 471.5 \\
$92-105$ & 96.9 & 36 & $117.6 \pm 16.9 \pm 8.3$ & 101.4 \\
$105-130$ & 118.4 & 68 & $76.1 \pm 7.8 \pm 3.7$ & 63.5 \\
$130-160$ & 148.2 & 70 & $34.0 \pm 5.0 \pm 2.3$ & 41.3 \\
$160-175$ & 167.1 & 82 & $33.5 \pm 3.6 \pm 2.1$ & 32.5 \\
\hline
\end{tabular}


number of selected events, $N_{\mathrm{ee}}$, corrected for the expected background, $N_{\mathrm{bg}}^{\mathrm{MC}}$, and the Monte Carlo prediction for Bhabha scattering, $N_{\mathrm{ee}}^{\mathrm{MC}}$ :

$\sigma\left(\left\langle\sqrt{s^{\prime}}\right\rangle\right)=\sigma_{\mathrm{ee}}^{\mathrm{Born}}\left(\left\langle\sqrt{s^{\prime}}\right\rangle\right) \frac{N_{\mathrm{ee}}-N_{\mathrm{bg}}^{\mathrm{MC}}}{N_{\mathrm{ee}}^{\mathrm{MC}}}$.

The value $\left\langle\sqrt{s^{\prime}}\right\rangle$ is the mean of the reconstructed effective centre-of-mass energies of the data in the
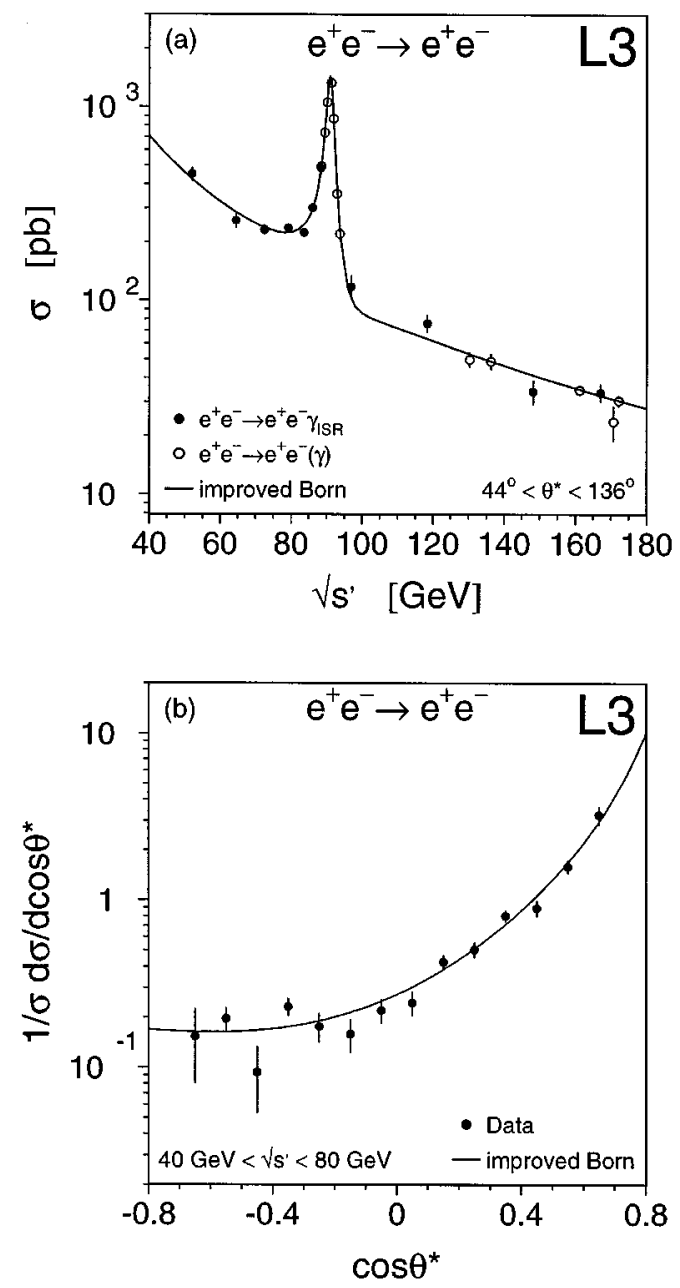

Fig. 4. (a) Measured total cross sections of Bhabha scattering. The theory prediction (solid line) is the result of the improved Born calculation. The results of this analysis, $\mathrm{e}^{+} \mathrm{e}^{-} \rightarrow \mathrm{e}^{+} \mathrm{e}^{-} \gamma_{\mathrm{ISR}}$, are shown as full dots. The open dots are the $\mathrm{L} 3$ results from the inclusive measurements at energies around and above the $\mathrm{Z}$ pole. They are corrected for the effect of initial-state photon radiation to correspond to this analysis. (b) The differential cross section of Bhabha scattering with $40 \mathrm{GeV}<\sqrt{s^{\prime}}<80 \mathrm{GeV}$. The theory prediction (solid line) is calculated at improved Born level. corresponding energy bin. The background contribution from tau-pair production amounts to $2.0 \%$ of the remaining sample. The background contributions from the processes $\mathrm{e}^{+} \mathrm{e}^{-} \rightarrow \mathrm{e}^{+} \mathrm{e}^{-} \mathrm{e}^{+} \mathrm{e}^{-}$and $\mathrm{e}^{+} \mathrm{e}^{-} \rightarrow$ $\gamma \gamma(\gamma)$ are found to be less than $0.1 \%$ and are therefore negligible.

The number of selected events, $N_{\mathrm{ee}}$, and the cross sections for the 11 different energy bins are listed in Table 2. The quoted systematic errors account for the limited Monte Carlo statistics. Other systematic errors are negligible.

The results are shown in Fig. 4a and compared to the improved Born calculation. The measurements are in good agreement with the theoretical prediction. Also shown are our inclusive cross section measurements near and above the Z-pole energy [14]. They are corrected for the effect of initial-state radiation using the program TOPAZ0.

\subsection{Differential cross section}

The scattering angle, $\theta^{*}$, of the electron, $\mathrm{e}^{-}$, in the centre-of-mass system is given by:

$\cos \theta^{*}=\frac{\sin \left(\theta_{\mathrm{e}^{+}}-\theta_{\mathrm{e}^{-}}\right)}{\sin \theta_{\mathrm{e}^{+}}+\sin \theta_{\mathrm{e}^{-}}}$.

Electron and positron are distinguished using the charge information from the central tracking system. Since Bhabha scattering is dominated by $t$-channel photon exchange for centre-of-mass energies well below the $Z$ resonance, the shape of the angular distribution is insensitive to the energy. Therefore, all events selected with an effective centre-of-mass energy between $40 \mathrm{GeV}$ and $80 \mathrm{GeV}$ are combined to measure the differential cross section. Each cross section point is determined by comparing the number of observed events with the Monte Carlo prediction:

$\frac{\mathrm{d} \sigma}{\mathrm{d} \cos \theta^{*}}=\frac{\mathrm{d} \sigma_{\mathrm{ee}}^{\text {Born }}}{\mathrm{d} \cos \theta^{*}} \cdot \frac{N_{\mathrm{ee}}-N_{\mathrm{bg}}^{\mathrm{MC}}}{N_{\mathrm{ec}}^{\mathrm{MC}}}$.

The results are shown in Fig. 4b. The preference for forward scattering of the $\mathrm{e}^{-}$due to the dominant 
$t$-channel exchange is clearly observed and in agreement with expectations.

\section{Compton scattering of quasi-real photons}

\subsection{Event selection}

In case of quasi-real Compton scattering one of the incoming beam electrons remains inside the beam pipe and is not detected. The energy depositions in the calorimeter are caused by the Compton scattered electron and photon.

For the selection of Compton scattering events both electron and photon are required to be observed within $|\cos \theta|<0.94$. The scattering angle in the centre-of-mass system of the $\gamma \mathrm{e}$ pair has to lie inside $\left|\cos \theta^{*}\right|<0.8$ to reduce the contribution from low angle Bhabha scattering.

The ratio $E_{1} / E_{1}^{a}$ is required to be greater than 0.7. All events containing a third electromagnetic cluster with an energy larger than $2 \mathrm{GeV}$ and an angular separation of more than $8^{\circ}$ from the identified electron are removed. To ensure the selection of quasi-real photons, the transverse momentum of the final state system has to be lower than $15 \%$ of the beam energy. This cut restricts the momentum transfer to the photon, suppressing the contribution from off-shell photons. The photons of the remaining sample have an average $Q^{2}$ between $2 \mathrm{GeV}^{2}$ and $9 \mathrm{GeV}^{2}$

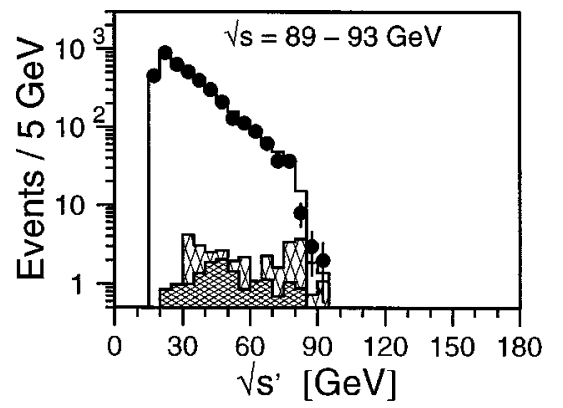

- Data

$\square \mathrm{MC} \gamma \mathrm{e} \rightarrow \gamma \mathrm{e}$

$\mathrm{MC} \mathrm{e}^{+} \mathrm{e}^{-} \rightarrow \mathrm{e}^{+} \mathrm{e}^{-}(\gamma)$

桨图 $M C \mathrm{e}^{+} \mathrm{e}^{-} \rightarrow \gamma \gamma(\gamma)$
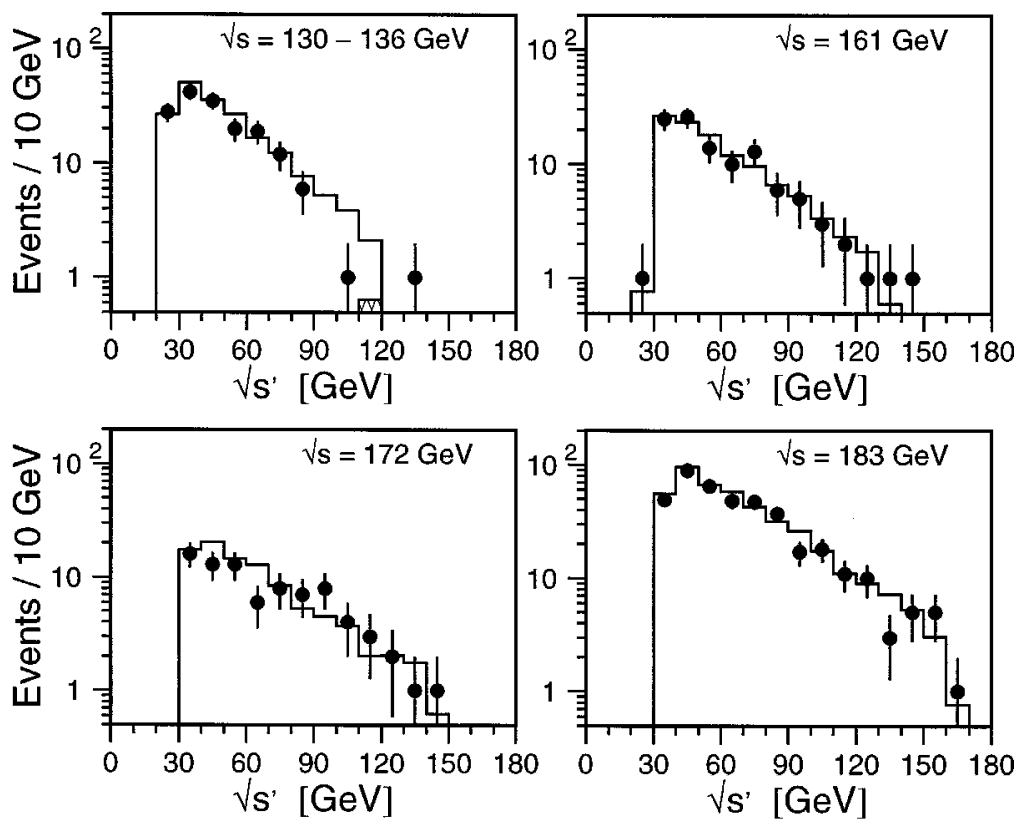

Fig. 5. The $\sqrt{s^{\prime}}$ spectra of Compton scattering for data and Monte Carlo simulation for signal and background are shown for different centre-of-mass energies. All selection cuts are applied. 
dependent on the centre-of-mass energy resulting in a small average virtuality $Q^{2} / s^{\prime}$ of the order $10^{-3}$.

Higher background contributions are expected when the effective centre-of-mass energy is close to $\sqrt{s}$ from either photon conversion in the reaction $\mathrm{e}^{+} \mathrm{e}^{-} \rightarrow \gamma \gamma(\gamma)$ in case that the two resulting tracks are not resolved or from Bhabha scattering if one track is lost. To remove these backgrounds the energy of the lower energetic cluster of the $\gamma \mathrm{e}$ pair, $E_{2}$, is required to be less than $85 \%$ of the beam energy. The distributions of $E_{2} / E_{\text {beam }}$ are shown in Fig. 2c and Fig. 2d. Fig. 5 shows the resulting $\sqrt{s^{\prime}}$-distributions for Compton scattering which are found to be in good agreement with the Monte Carlo expectations. The total cross sections at reduced centre-of-mass energies are derived from the combined distributions.

\subsection{Total cross sections}

In total 4641 candidates for Compton scattering are selected from the data sample. The total cross sections are calculated as in the analysis of Bhabha scattering (Eq. (4)) rescaling the theoretical cross section, $\sigma_{\gamma \mathrm{e}}^{\mathrm{QED}}$, determined from the QED calculation. The background contributions from Bhabha scattering and from the process $\mathrm{e}^{+} \mathrm{e}^{-} \rightarrow \gamma \gamma(\gamma)$ are found to be less than $0.5 \%$ of the expected events.

Table 3 contains the numbers of selected events, $N_{\gamma \mathrm{e}}$, and the measured total cross sections of Compton scattering for 10 different $\sqrt{s^{\prime}}$ bins. The quoted systematic error is dominated by the limited Monte
Carlo statistics. Fig. 6a shows the results compared with the QED prediction for the Compton scattering cross section. The measurements are in good agreement with the theoretical prediction.

\subsection{Differential cross section}

The scattering angle, $\theta^{*}$, of the electron in the centre-of-mass system is given in the case of $\gamma \mathrm{e}$ scattering by:

$\cos \theta^{*}=\frac{\sin \left(\theta_{\gamma}-\theta_{\mathrm{e}^{-}}\right)}{\sin \theta_{\gamma}+\sin \theta_{\mathrm{e}^{-}}}$.

Since the polar angles $\theta_{\gamma}$ and $\theta_{\mathrm{e}^{-}}$are measured with respect to the direction of the incoming electron $\left(\mathrm{e}^{-}\right)$, the scattering angle is defined by $\pi-\theta^{*}$ in case of $\gamma \mathrm{e}^{+}$scattering.

Combining all energy bins from $20 \mathrm{GeV}$ to $80 \mathrm{GeV}$ the differential cross section of Compton scattering is derived. The results are shown in Fig. 6b. The measurement exhibits the preference for backward scattering in the centre-of-mass system, which is characteristic for unpolarized Compton scattering.

\section{Production of single excited electrons}

Models including excited leptons [15] predict that the production of excited electrons in $\mathrm{e}^{+} \mathrm{e}^{-}$collisions is extremely peaked in the forward direction. Therefore, the electron in the process $\mathrm{e}^{+} \mathrm{e}^{-} \rightarrow \mathrm{ee}^{\star}$

Table 3

Number of selected $\gamma \mathrm{e} \rightarrow \gamma \mathrm{e}$ events, $N_{\gamma \mathrm{e}}$, for the various ranges of $\sqrt{s^{\prime}}$ and their average $\left\langle\sqrt{s^{\prime}}\right\rangle$. The corresponding measured cross sections, $\sigma_{\gamma \mathrm{e}}$, and their statistical and systematic errors are listed and compared to the QED cross sections, $\sigma_{\gamma \mathrm{e}}^{\mathrm{QED}}$

\begin{tabular}{lcccc}
\hline$\sqrt{s^{\prime}}[\mathrm{GeV}]$ & $\left\langle\sqrt{s^{\prime}}\right\rangle[\mathrm{GeV}]$ & $N_{\gamma \mathrm{e}}$ & $\sigma_{\gamma \mathrm{e}} \pm(\mathrm{stat}) \pm(\mathrm{syst})[\mathrm{pb}]$ & $\sigma_{\gamma \mathrm{e}}^{\mathrm{QED}}[\mathrm{pb}]$ \\
\hline$<25$ & 21.0 & 1346 & $771.2 \pm 20.8 \pm 5.8$ & 764.8 \\
$25-35$ & 29.8 & 1225 & $370.6 \pm 10.9 \pm 2.9$ & 381.1 \\
$35-45$ & 39.7 & 868 & $210.7 \pm 7.3 \pm 2.1$ & 215.0 \\
$45-55$ & 49.5 & 471 & $125.0 \pm 6.4 \pm 1.8$ & 138.3 \\
$55-65$ & 59.5 & 302 & $95.3 \pm 5.5 \pm 1.7$ & 95.5 \\
$65-75$ & 69.6 & 184 & $65.3 \pm 5.2 \pm 1.7$ & 69.8 \\
$75-85$ & 79.1 & 111 & $57.6 \pm 5.1 \pm 1.9$ & 54.1 \\
$85-100$ & 91.0 & 60 & $37.5 \pm 5.3 \pm 2.0$ & 40.9 \\
$100-120$ & 109.4 & 42 & $26.5 \pm 4.4 \pm 1.7$ & 28.3 \\
$120-170$ & 136.0 & 32 & $18.5 \pm 3.2 \pm 1.4$ & 18.3 \\
\hline
\end{tabular}



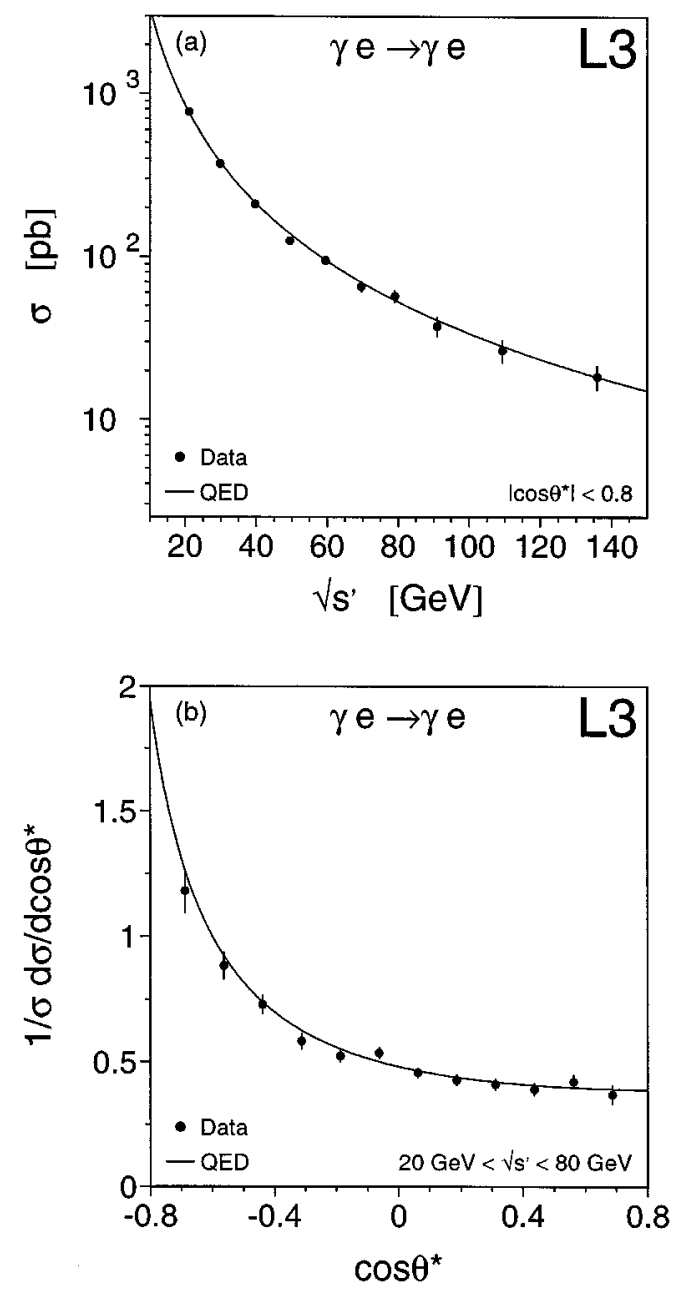

Fig. 6. (a) Measured total cross sections of Compton scattering inside the angular range $\left|\cos \theta^{*}\right|<0.8$ as a function of the effective centre-of-mass energy $\sqrt{s^{\prime}}$. The solid line shows the QED prediction. (b) The differential cross section of Compton scattering as a function of the scattering angle in the $\gamma \mathrm{e}$ rest frame. The solid line shows the QED prediction.

can be lost in the beam pipe whereas the $\gamma$ e decay products of the excited electron would be seen inside the detector. Consequently, an exclusion limit on the production of excited electrons can be derived from the measurement of Compton scattering.

Within the theoretical model for excited leptons, the coupling $\mathrm{e}^{\star} \mathrm{e} \gamma$ is described by one dimensionless parameter $\lambda[16]$. The cross section for the production of an excited electron $e^{\star}$ in electron-pho- ton collisions, decaying into an $\gamma \mathrm{e}$ pair is then given by [17]:

$$
\begin{aligned}
& \sigma\left(\mathrm{e} \gamma \rightarrow \mathrm{e}^{\star} \rightarrow \mathrm{e} \gamma\right)\left(m_{\mathrm{e}^{\star}}\right) \\
& =\frac{4 \pi^{2} \alpha \lambda^{2}}{m_{\mathrm{e}^{\star}}^{2}} \frac{\Gamma\left(\mathrm{e}^{\star} \rightarrow \mathrm{e} \gamma\right)}{\Gamma_{\mathrm{e}^{\star}}} .
\end{aligned}
$$

This expression is valid assuming a narrow width for the excited electron. The partial width for the radiative decay is given by [17]:

$\Gamma\left(\mathrm{e}^{\star} \rightarrow \mathrm{e} \gamma\right)=\frac{1}{2} \lambda^{2} \alpha m_{\mathrm{e}^{\star}}$.

The total width, $\Gamma_{\mathrm{e}^{\star}}$, also takes into account the partial widths for the decays $\mathrm{e}^{\star} \rightarrow \nu \mathrm{W}$ and $\mathrm{e}^{\star} \rightarrow \mathrm{eZ}$ for masses $m_{\mathrm{e}^{\star}}$ above the masses of the $\mathrm{W}$ and $\mathrm{Z}$ bosons [18].

Within an interval $m_{\mathrm{e}^{\star}} \pm \Delta$, the number of observed $\gamma$ e pairs and the expected contribution from Compton scattering, $N_{\gamma \mathrm{e}}$, are determined. These numbers are used to calculate the $95 \%$ confidence level upper limit for the number of events that could come from such a narrow $\mathrm{e}^{\star}$ resonance, $N_{\mathrm{e}} \star$. The interval width $\Delta$ is chosen as twice the invariant mass resolution of typically $1 \%$. Taking into account the expected angular distribution in the $\mathrm{e}^{\star}$ rest frame [15],

$$
\frac{1}{\sigma} \frac{\mathrm{d} \sigma}{\mathrm{d} \cos \theta}=\frac{1+\cos \theta}{2},
$$

the upper limit for the coupling $\lambda$ is determined by satisfying the inequality

$$
\begin{aligned}
\frac{N_{\mathrm{e}^{\star}}}{N_{\gamma \mathrm{e}}} \sigma_{\gamma \mathrm{e}}^{\mathrm{QED}}\left(m_{\mathrm{e}^{\star}}\right)> & \frac{\epsilon_{\mathrm{e}^{\star}}}{\epsilon_{\gamma \mathrm{e}}} \frac{\pi^{2} \alpha \lambda^{2}}{2 m_{\mathrm{e}^{\star} \Delta}} \\
& \times\left(c_{\max }-c_{\min }+\frac{c_{\max }^{2}-c_{\min }^{2}}{2}\right) \\
& \times \frac{\Gamma\left(\mathrm{e}^{\star} \rightarrow \mathrm{e} \gamma\right)}{\Gamma_{\mathrm{e}^{\star}}} .
\end{aligned}
$$

This uses the QED prediction for the Compton scattering cross section, $\sigma_{\gamma \mathrm{e}}^{\mathrm{QED}}$, at an effective centre-ofmass energy given by the mass $m_{\mathrm{e}^{\star}}$. The limit on the coupling parameter $\lambda$ takes into account the efficiencies $\epsilon_{\mathrm{e}^{\star}}$ and $\epsilon_{\gamma \mathrm{e}}$ and the acceptance cuts in the $\mathrm{e}^{\star}$ rest frame, $c_{\min }<\cos \theta^{*}<c_{\max }$.

The result is shown in Fig. 7. A comparable limit has been derived from our dedicated search for excited electrons based on data collected at energies 


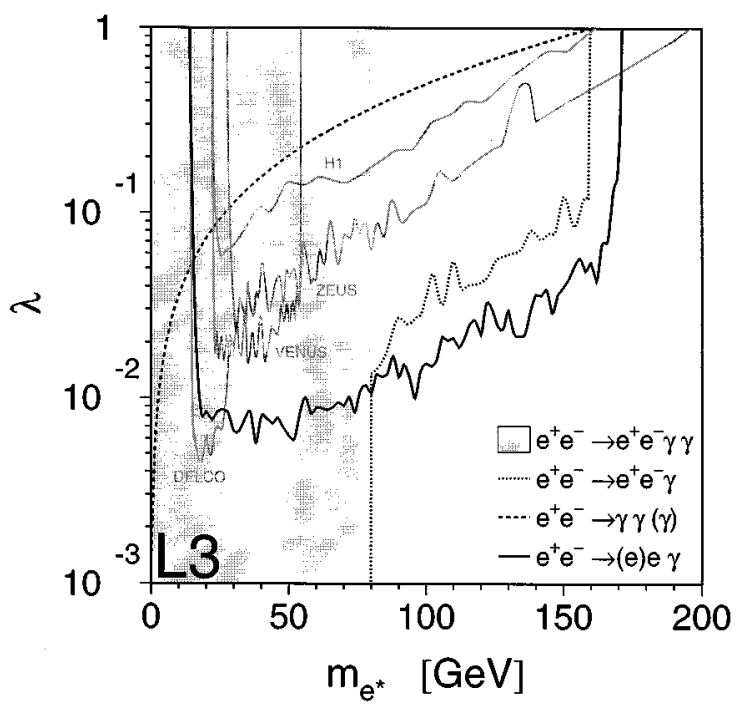

Fig. 7. The upper limit of the coupling constant $\lambda$ at $95 \%$ confidence level as a function of $m_{\mathrm{e}}$, derived from the measurement of Compton scattering (solid black line). The dashed line shows the analogous result from our previous analysis of the process $\mathrm{e}^{+} \mathrm{e}^{-} \rightarrow \gamma \gamma(\gamma)$, based on the data recorded at centre-ofmass energies between $161 \mathrm{GeV}$ and $172 \mathrm{GeV}$ [20]. Using data collected at energies up to $161 \mathrm{GeV}$, excited electrons with masses up to $79.7 \mathrm{GeV}$ at $95 \%$ confidence level are excluded from our analysis of the pair production $\mathrm{e}^{\star} \mathrm{e}^{\star}$ in the channel ee $\gamma \gamma$ (shaded area), and an upper limit on the coupling $\lambda$ is also derived from our search for the single production of an excited electron (dotted line) [19]. The results from other collider experiments are included as indicated $[21,22]$.

up to $161 \mathrm{GeV}$ [19]. For comparison, the upper limit for the $\mathrm{e}^{\star} \mathrm{e} \gamma$ coupling from our analysis of the QED reaction $\mathrm{e}^{+} \mathrm{e}^{-} \rightarrow \gamma \gamma(\gamma)$ is also shown [20]. From our analysis of the pair production $\mathrm{e}^{\star} \mathrm{e}^{\star}$ in the channel ee $\gamma \gamma$, a lower mass limit of $79.7 \mathrm{GeV}$ at $95 \%$ confidence level is derived [19]. The results from previous experiments using the process of quasi-real Compton scattering at $\mathrm{e}^{+} \mathrm{e}^{-}$colliders [21] and the upper limits from the experiments at the HERA ep collider [22] are included for comparison. Other searches for excited electrons at LEP are reported in Ref. [23].

\section{Conclusions}

The effect of initial-state radiation in the reaction $\mathrm{e}^{+} \mathrm{e}^{-} \rightarrow \mathrm{e}^{+} \mathrm{e}^{-}(\gamma)$ is studied. The selected events are used to measure Bhabha scattering at effective centre-of-mass energies between $50 \mathrm{GeV}$ and $170 \mathrm{GeV}$. The measurements show good agreement with the Standard Model predictions.

Electron-photon events are identified as Compton scattering of quasi-real photons. We measure this process in the centre-of-mass energy range from 20 $\mathrm{GeV}$ to $140 \mathrm{GeV}$ which is the highest energy at which Compton scattering has been studied so far. The total rate and the observed differential cross sections are in good agreement with the theoretical expectations.

An upper limit for a hypothetical coupling $\mathrm{e}^{\star} \mathrm{e} \gamma$ as a function of $m_{e^{\star}}$ is derived from the measurement of Compton scattering. No indications for the existence of an excited electron are found in the data. The $95 \%$ confidence level limit on the coupling parameter $\lambda$ is of the order $10^{-2}$ to $10^{-1}$ for the mass region $20 \mathrm{GeV}<m_{\mathrm{e}^{\star}}<170 \mathrm{GeV}$.

\section{Acknowledgements}

We wish to express our gratitude to the CERN accelerator divisions for the excellent performance of the LEP machine. We acknowledge the contributions of all the engineers and technicians who have participated in the construction and maintenance of this experiment.

\section{References}

[1] ALEPH Collaboration, D. Buskulic et al., Z. Phys. C 62 (1994) 539; ALEPH Collaboration, D. Buskulic et al., Phys. Lett. B 378 (1996) 373; DELPHI Collaboration, P. Abreu et al., Nucl. Phys. B 418 (1994) 403; L3 Collaboration, M Acciarri et al., Z. Phys. C 62 (1994) 551; L3 Collaboration, M. Acciarri et al., Phys. Lett. B 370 (1996) 195; L3 Collaboration, M. Acciarri et al., Phys. Lett. B 407 (1997) 361; OPAL Collaboration, R. Akers et al., Z. Phys. C 61 (1994) 19; OPAL Collaboration, K. Ackerstaff et al., Phys. Lett. B 376 (1996) 232; OPAL Collaboration, K. Ackerstaff et al., Euro. Phys. Journal C 2 (1998) 441.

[2] HRS Collaboration, M. Derrick et al., Phys. Rev. D 34 (1986) 3286; MAC Collaboration, E. Fernandez et al., Phys. Rev. D 35 (1987) 10; Mark II Collaboration, D. Karlen et al., Phys. Rev. D 39 (1989) 1861; CELLO Collaboration, H.J Behrend et al., Phys. Lett. B 103 (1981) 148; JADE Collaboration, W. Bartel et al., Z. Phys. C 30 (1986) 371; MARK J Collaboration, B. Adeva et al., Phys. Rep. 109 (1984) 131; 
PLUTO Collaboration, C. Berger et al., Z. Phys. C 27 (1985) 341; TASSO Collaboration, W. Braunschweig et al., Z. Phys. C 37 (1988) 171; C. Rosenfeld, Recent results from AMY, Talk presented at the XXIIIrd Rencontres de Moriond, Les Arcs, France, 1988; T. Ishii, New results from TOPAZ on leptonic processes, Talk presented at the XXIIIrd Rencontres de Moriond, Les Arcs, France, 1988; VENUS Collaboration, T. Arima et al., Phys. Rev. D 55 (1997) 19.

[3] ALEPH Collaboration, R. Barate et al., Phys. Lett. B 399 (1997) 329; DELPHI Collaboration, P. Abreu et al., Z. Phys. C 75 (1997) 581; L3 Collaboration, M. Acciarri et al., Phys. Lett. B 374 (1996) 331; OPAL Collaboration, P.D. Acton et al., Phys. Lett. B 273 (1991) 338.

[4] M. Caffo, E. Rimidi et al., in: G. Altarelli, R. Kleiss, C. Verzegnassi (Eds.), Z Physics at LEP 1, Report CERN 89-08, 1989, vol. 1, p. 171.

[5] A. Courau, P. Kessler, Phys. Rev. D 33 (1986) 2024.

[6] L3 Collaboration, B. Adeva et al., Nucl. Inst. Meth. A 289 (1990) 35; M. Chemarin et al., Nucl. Inst. Meth. A 349 (1994) 345; M. Acciari et al., Nucl. Inst. Meth. A 351 (1994) 300; G. Basti et al., Nucl. Inst. Meth. A 374 (1996) 293; I. Brock et al., Nucl. Inst. Meth. A 381 (1996) 236; A. Adam et al., Nucl. Inst. Meth. A 383 (1996) 342.

[7] J.H. Field, T. Riemann, Comp. Phys. Comm. 94 (1996) 53. [8] D. Karlen, Nucl. Phys. B 289 (1987) 23.

[9] S. Jadach, Z. Wąs, Comp. Phys. Comm. 79 (1994) 503.

[10] F.A. Berends, R. Kleiss, Nucl. Phys. B 186 (1981) 22.

[11] F.A. Berends, R. Kleiss, Nucl. Phys. B 253 (1985) 441.

[12] The L3 detector simulation is based on GEANT Vers. 3.15. R. Brun et al., GEANT 3, CERN-DD/EE/84-1 (Revised)
1987. The GHEISHA program (H. Fesefeldt, RWTH Aachen Report PITHA 85/02 (1985)) is used to simulate hadronic interactions.

[13] TOPAZ0 version 2.0 is used. G. Montagna, F. Piccinini, O. Nicrosini, G. Passarino, R. Pittau, Nucl. Phys. B 401 (1993) 3; Comp. Phys. Comm. 76 (1993) 328.

[14] L3 Collaboration, M. Acciarri et al., Z. Phys. C 62 (1994) 551; Phys. Lett. B 370 (1996) 195; B 407 (1997) 361.

[15] K. Hagiwara, D. Zeppenfeld, S. Komamiya, Z. Phys. C 29 (1985) 115.

[16] F. Low, Phys. Rev. Lett. 14 (1965) 238; H. Terazawa et al., Phys. Lett. B 112 (1982) 387.

[17] F.M. Renard, Z. Phys. C 14 (1982) 209.

[18] F. Boudjema, A. Djouadi, J.L. Kneur, Z. Phys. C 57 (1993) 425.

[19] L3 Collaboration, M. Acciarri et al., Phys. Lett. B 401 (1997) 139.

[20] L3 Collaboration, M. Acciarri et al., Phys. Lett. B 413 (1997) 159.

[21] CELLO Collaboration, H.J. Behrend et al., Phys. Lett. B 168 (1986) 420; DELCO Collaboration, G. Bonneaud et al., Phys. Lett. B 177 (1986) 109; VENUS Collaboration, K. Abe et al., Phys. Lett. B 213 (1988) 400.

[22] H1 Collaboration, S. Aid et al., Nucl. Phys. B 483 (1997) 44; ZEUS Collaboration, J. Breitweg et al., Z. Phys. C 76 (1997) 631.

[23] ALEPH Collaboration, D. Buskulic et al., Phys. Lett. B 385 (1996) 445; DELPHI Collaboration, P. Abreu et al., Phys. Lett. B 393 (1997) 245; OPAL Collaboration, K. Ackerstaff et al., Eur. Phys. J. C 1 (1998) 45. 